M. Bijarimi ${ }^{*}$, A. Syuhada ${ }^{1}$, N. Zulaini ${ }^{1}$, N. Shahadah ${ }^{1}$, W. Alhadadi ${ }^{1}$, M. N. Ahmad ${ }^{2}$, A. Ramli ${ }^{1}$, E. Normaya ${ }^{2}$

${ }^{1}$ Faculty of Chemical \& Natural Resources Engineering, Universiti Malaysia Pahang, Gambang, Pahang, Malaysia

${ }^{2}$ Experimental and Theoretical Research Laboratory, Department of Chemistry, Kulliyyah of Science, International Islamic University Malaysia, Kuantan, Pahang, Malaysia

\title{
Poly(lactic acid)/Acrylonitrile Butadiene Styrene Nanocomposites with Hybrid Graphene Nanoplatelet/Organomontmorillonite: Effect of Processing Temperatures
}

This work reports the preparation and characterization of poly(lactic) acid/acrylonitrile butadiene styrene/graphene nanoplatelets/Cloisite C20A montmorillonite (PLA/ABS/GnP/C20A) nanocomposites via melt blending. The clay is hybridized with graphene to increase its dispersion in the polymer matrix. The melt processing temperatures play a vital role in the properties of the resulting nanocomposites in dictating the extent of thermal stability and dispersion of the fillers. The hybrid nanocomposites were characterized for stress-strain, thermal, chemical, and morphological properties. The findings were that there was an increase in the mechanical properties in terms of tensile strength and Young's modulus with the PLA/ABS/GnP/C2OA at the high-temperature profile having the highest values of 43.1 MPa and $2533 \mathrm{MPa}$. The elongation at break increases slightly, due to the brittle properties of GnP. It was found that the dispersion of the fillers increased with increasing temperature profiles, as revealed by the morphological analysis by scanning electron microscopy (SEM) and transmission electron microscopy (TEM). The void size was also observed to be smaller and more homogenous with increasing temperature. However, in terms of thermal degradation analysis, the addition of fillers increases its thermal stability as the decomposition onset temperature increases by $22.5^{\circ} \mathrm{C}$.

\section{Introduction}

Nowadays, the search for polymers that are biodegradable and benign to the environment has been exploited by tailoring the existing commodity polymers. Poly(lactic acid) is a bioplastic made using renewable biomass known as PLA. Renewable resources such as starch and sugar are one of the sources. PLA

\footnotetext{
* Mail address: Mohd Bijarimi Mat Piah, Faculty of Chemical \& Natural Resources Engineering, Universiti Malaysia Pahang, Lebuhraya Tun Razak, 26300 Gambang, Pahang, Malaysia

E-mail: bijarimi@ump.edu.my
}

is an eco-friendly, biocompatible, and renewable polymer that can be processed using conventional processing equipment. Typically, the use of non-degradable plastics for consumer products and engineering goods has created a substantial dumping problem. As such, polymers from renewable sources such as PLA are on the threshold of gaining popularity. PLA has been extensively blended with other polymers to modify its inherent brittleness, mainly rubber toughening, as demonstrated by previous research (Nofar et al., 2019). However, PLA is very brittle to be used commercially, and toughness modification is typically required. Various strategies have been employed to toughen PLA, such as blending with plasticizers' and rubber/ plastic blends (Bijarimi et al., 2017; 2014, Bitinis et al., 2012; Pluta et al., 2006). In many cases, the strength and modulus of the toughened PLA are much lower than those of neat PLA. PLA also has high strength and modulus, insufficient toughness, and low thermal stability.

Acrylonitrile butadiene styrene (ABS) is a tough material and suitable for toughening brittle PLA (Vadori et al., 2016). ABS is composed of acrylonitrile, butadiene, and styrene; it has strength and rigidity. The toughness in ABS is given by the rubbery part of polybutadiene in the block copolymer (Vadori et al., 2017). ABS has been widely used for electrical and electronics goods, automotive parts, and household appliances. Recently, the use of ABS for 3D printing has increased tremendously, as many prototype developments require the aid of 3D printer prior to commercialization. In ABS/PLA blends, part of the ABS could be substituted with PLA in an effort to increase the content of the eco-friendly polymer. Li and Shimizu (2009) reported that the incompatibilized blends of PLLA and ABS exhibited poor mechanical properties with low elongation at break and impact strength. However, they found that a reactive styrene/acrylonitrile/glycidyl methacrylate copolymer (SAN-GMA) by incorporating ethyl triphenylphosphonium bromide (ETPB) as the catalyst, SAN-GMA was an effective reactive compatibilizer for PLLA/ABS. The improvement in impact strength and elongation at break was attributed to the dispersion of rubber particles in the blend. Choe et al. (2014) 
investigated the mechanical properties of acrylonitrile-butadiene-styrene copolymer/poly(l-lactic acid) (70/30) wt.\% blends and their composites. They found that the SAN-gGMA random copolymer was an effective compatibilizer for the ABS/PLA blends. In another work by Dong et al. (2015) PLLA/ABS blend was compatibilized by a reactive comb (RC) polymer, i.e. a poly-(methyl methacrylate) (PMMA) backbone, which was distributed randomly along the backbone. Their results showed that the improvement in toughness was most significant at the weight ratio of PLLA/ABS (50/ $50 \%$ ). In an effort to improve the toughness of PLLA, Wu et al. (2015) incorporated $6.0 \mathrm{wt} . \%$ ABS copolymer particles in a poly(L-lactide) (PLLA) matrix. They reported that the ABS copolymer increased the elongation at break and impact strength as compared to neat PLLA. Vadori et al. (2016) explored polymer blends containing poly(lactic acid) (PLA) and acrylonitrile butadiene styrene (ABS) $(50 / 50 \%)$. They found that acrylic copolymers and chain extender worked to synergistically increase the impact strength.

Polymer nanocomposites typically contain nanofillers such as organomontmorillonite or graphene. Montmorillonite (MMT) is a cost-effective additive in polymers and currently the most researched filler for polymer composites. The use of MMT and graphene individually as nanofiller in the polymer matrix has been well established and available in the literature (Alexandre et al., 2000; Arroyo et al., 2010; Bijarimi et al., 2019; 2020; Coiai et al., 2017; Gonçalves et al., 2016; Jalalvandi et al., 2015; Keramati et al., 2016; Li et al., 2017; Manafi et al., 2014; Pinto et al., 2016; Sabzi et al., 2015; Scaffaro et al., 2016; 2017; Sharma et al., 2018; Young et al., 2018). Nevertheless, there is a limitation for polymer nanocomposites based on MMT alone, such as brittleness and thermal conductivity. On the other hand, graphene is considered an ideal nano-reinforcement for multifunctional polymer nanocomposites owing to its extraordinary thermomechanical and electrical properties (Fu et al., 2014). Even at a low load quantity of graphene, it can improve the overall mechanical properties of the polymer. Besides, graphene is expected to have significant characteristics such as high thermal conductivity, outstanding electronic transport properties, and exceptional mechanical properties. Polymer nanocomposites based on hybrid organomontmorillonite and graphene nanoplatelets $(\mathrm{GnP})$ offer a new class of material by taking advantage of the performance of MMT and $\mathrm{GnP}$ as individual nanofillers. The synergistic effect of MMT and graphene functionalized epoxy in PLA blends was reported by Bouakaz et al. (2015). Their results showed that the enhancement of dispersion and exfoliation of two nanofillers (organomontmorillonite and graphene) in ternary PLA nanocomposites is ascribed to a right combination of repulsive and attractive interactions between the C15A-graphene and C30B-graphene systems. It is very interesting to evaluate the hybridization effect of MMT/graphene for other types of blend systems such as PLA/ABS blends. In this work, part of the ABS was substituted with PLA in the ABS matrix in order to develop an ABS blend with $20 \%$ eco-friendly material. In addition, it was expected that the rigidity of the ABS blend could be enhanced by the presence of PLA, as a dispersed phase. The aim of this work was to prepare and char- acterize PLA/ABS with a hybrid of graphene and MMT as nanofillers in terms of mechanical, thermal, chemical, and morphological properties. The focus of the study is the effect of processing temperatures for three ranges of temperature profiles i.e. low, medium, and high temperatures.

\section{Materials and Methods}

\subsection{Materials}

Poly(lactic acid) of NatureWorks Ingeo Biopolymer 3251D grade supplied by Kinetic Chemicals (M) Sdn. Bhd., Kuala Lumpur, Malaysia, was the thermoplastic resin used in this study. It has a density of $1.24 \mathrm{~g} / \mathrm{cm}^{3}$ and a melt flow index of $35 \mathrm{~g} / 10 \mathrm{~min}\left(190^{\circ} \mathrm{C} / 2.16 \mathrm{~kg}\right)$ and a melting temperature between 155 to $170^{\circ} \mathrm{C}$. Acrylonitrile butadiene styrene (ABS) Toyolac 700314 with a density of $1.05 \mathrm{~g} / \mathrm{cm}^{3}$ and melt flow index of $25 \mathrm{~g} / 10 \mathrm{~min} .\left(220^{\circ} \mathrm{C} / 10 \mathrm{~kg}\right)$ acquired from Toray Plastic, Penang, Malaysia, has a glass transition temperature of $103^{\circ} \mathrm{C}$ with no exact melting temperature due to its amorphous nature. Graphene nanoplatelets ( $\mathrm{GnPs})$ are named graphitic nano particles which have layered arrangements. They involve combined 2D graphene sheets stacked with van der Waals forces. Grade "M" GnP was found from XG Sciences (Lansing, Michigan, USA) with a density of $2.2 \mathrm{~g} / \mathrm{cm}^{3}$, average width between 6 to $8 \mathrm{~nm}$, and superficial area between 120 to $150 \mathrm{~m}^{2} / \mathrm{g}$. Natural montmorillonite modified with a quaternary ammonium salt (Cloisite C20A from Southern Clay Inc., Gonzales, USA) was used in this study. Cloisite C20A is known as an additive for plastics and rubbers to improve various physical properties such as reinforcement, synergistic flame retardancy, and barriers. Other chemicals were used as received.

\subsection{Preparation of ABS/PLA and Nanocomposites}

The compositions of PLA/ABS binary blend and GnP/MMT nanofillers are given in Table 1. The first step of the melt blending process was the production of the masterbatch, which was carried out using a twin-screw extruder (model Thermo Electron Prism Euro, Thermo Fisher Scientific Inc., Waltham, Massachusetts, USA) with a screw diameter of $16 \mathrm{~mm}$ and L/ $\mathrm{D}$ ratio of 40 . The extrusion screw speed was $50 \mathrm{~min}^{-1}$ with a residence time of $180 \mathrm{~s}$, and the temperature profiles between 150 and $220^{\circ} \mathrm{C}$. The PLA and ABS resins were dried in an oven at $50^{\circ} \mathrm{C}$ for $12 \mathrm{~h}$. The PLA/ABS pellets and the $\mathrm{GnP} /$ OMMT were fed simultaneously into the extruder.

The extrudates were dried and collected and then molded at $180^{\circ} \mathrm{C}$ under $45 \mathrm{MPa}$ of pressure for $10 \mathrm{~min}$ using a hot press to produce a sheet measuring $150 \mathrm{~mm}$ width $\times 125 \mathrm{~mm}$ length with $1 \mathrm{~mm}$ thickness.

For the effect of processing temperatures, there are three profiles employed i.e., low temperature (LT), medium temperature (MT) and high temperature (HT). The temperature details for each zone and profiles are given in Table 2. 


\begin{tabular}{|c|c|c|}
\hline Designation & Compositions & Parts \\
\hline PLA & PLA & 100 \\
PLA/ABS & PLA/ABS & $20 / 80$ \\
PLA/ABS/GnP/C20A & PLA/ABS/GnP/C20A & $20 / 80 / 1 / 1$ \\
\hline
\end{tabular}

Table 1. Sample designation and composition

\begin{tabular}{|c|c|c|c|c|c|c|}
\hline Twin screw extruder zone & 1 & 2 & 3 & 4 & 5 & Die \\
\hline Low temperature profile $(\mathrm{LT})\left[{ }^{\circ} \mathrm{C}\right]$ & 190 & 180 & 180 & 175 & 172 & 168 \\
\hline Medium temperature profile $(\mathrm{MT})\left[{ }^{\circ} \mathrm{C}\right]$ & 200 & 190 & 190 & 180 & 175 & 168 \\
\hline High temperature profile $(\mathrm{HT})\left[{ }^{\circ} \mathrm{C}\right]$ & 210 & 200 & 200 & 190 & 180 & 168 \\
\hline
\end{tabular}

Table 2. Temperature profile of PLA/ABS/and PLA/ABS/GnP/MMT nanocomposites

\subsection{Mechanical Testing}

All blend compositions were tested and compared for their mechanical properties. The tensile test was carried out according to ASTM D638 using an universal testing machine (model AG-X, Shimadzu, Kyoto, Japan) under ambient conditions with crosshead speed of $50 \mathrm{~mm} \mathrm{~min}{ }^{-1}$. The tensile properties that are determined are the stress at a peak, elongation at break, Young's modulus, and maximum force load. Toughness was calculated from the areas under the curves of stress-strain. The stress-strain values are taken from an average of five specimens.

\subsection{Morphological Analysis}

The morphological characteristics were investigated via scanning electron microscopy (SEM). The morphological characteristics of the cross-sectional area of the fracture specimens resulting from the tensile test was performed using a scanning electron microscope (model Quanta 450, FEI, Hillsboro, USA) in low vacuum mode at $5.0 \mathrm{kV}$. Platinum was sputtercoated on the fracture surface before microscopic observation.

Transmission electron microscopy (TEM) morphology analysis was also carried out. Samples for TEM were trimmed into a trapezoidal shape of $1 \mathrm{~mm}^{2}$ blocks from the tensile specimen. The samples were then mounted onto an ultramicrotome (model EM UC7, Leica, Wetzlar, Germany) for sectioning with the temperature set at $-100^{\circ} \mathrm{C}$. A copper grid with propanol was used to pick up the sections from the frozen chamber and deposited onto a copper grid. The grid was then viewed under TEM (model Tecnai $\mathrm{G}^{2}$ 20, FEI, Hillsboro, USA) at 40 to $200 \mathrm{kV}$. Subsequently, the micrographs were processed using imaging system software.

\subsection{Fourier Transform Infrared (FTIR)}

Fourier transform infrared (FTIR) was used to monitor the chemical changes after blending. A FTIR spectrophotometer (model Nicolet iS5, Perkin-Elmer, Waltham, Massachusetts, USA) was used on the specimens to study the Fourier transform infrared - attenuated total reflectance (FT-ATR). Spectrum $400 \mathrm{FT}$-IR and a spectrometer with $4 \mathrm{~cm}^{-1}$ resolution and 10 scans were used to record the infrared (IR) spectra. The absorbance mode of all the spectra was recorded in the 4000 to $500 \mathrm{~cm}^{-1}$ region.

\subsection{Differential Scanning Calorimetry (DSC)}

Differential scanning calorimetry (DSC) was used to study the crystallization behavior of the blend components. The DSC measurements on specimens of 1 to $3 \mathrm{mg}$ were taken using a DSC214 apparatus (Netzsch, Selb, Germany). The specimens were tested from $-100{ }^{\circ} \mathrm{C}$ to $300^{\circ} \mathrm{C}$ at $10^{\circ} \mathrm{C} / \mathrm{min}$ scan rate. Liquid nitrogen was used to bring the temperature of the sample to $-100{ }^{\circ} \mathrm{C}$, and then nitrogen gas was used to purge the sample. The glass transition temperature, crystallization temperature, and melting temperature were analyzed.

\subsection{Thermogravimetric Analysis (TGA)}

Thermal degradation was evaluated using thermogravimetric analysis (TGA). The measurements were made of the change in mass of the specimen during heating as a function of time, and/or temperature. The decomposition of mass is due to chemical reactions or physical changes during heating. The evaluation of the thermal stability of PLA and blends was carried out by a STA7000 apparatus (Hitachi, Tokyo, Japan). Samples of $5 \pm 1 \mathrm{mg}$ were placed into a sample crucible (alumina). The reference alumina crucible was left empty. Samples were heated from ambient temperature to $900{ }^{\circ} \mathrm{C}$ at $10^{\circ} \mathrm{C} / \mathrm{min}$ scanning rate under a nitrogen atmosphere. The temperature, weight derivative and heat flow of the samples were recorded. 


\section{Result and Discussion}

\subsection{Mechanical Properties}

Table 3 summarizes the mechanical properties of the PLA, ABS, PLA/ABS and PLA/ABS/GnP/C20A nanocomposites. The reduction in the mechanical properties of the nanocomposites in comparison with the neat polymers is expected because it involves physical blends. In terms of mechanical properties across the different nanocomposites, it was expected that the tensile strength, elongation at break, and Young's modulus should increase with the increase in temperature. From Fig. 1, it is evident that PLA/ABS/GnP/C20A HT show the highest value in the stress-strain curves.

In Figure 2A, the tensile strength, as represented by the stress at the peak, shows that PLA/ABS/GnP/C20A has better performance in comparison with the PLA/ABS nanocomposites. There is an increase of $20 \%$ and $15 \%$ when comparing PLA/ABS and PLA/ABS/GnP/C20A at MT and HT profiles, respectively. The effect of the addition of nanofillers has been demonstrated in other studies, such as findings by Fukushima et al. (2010) and Boakaz et al. (2015). Besides, the tensile strength was also observed to increase with increasing temperature. This is because an increase in temperature increases the kinetic energy required to overcome the immense van der Waals forces between the GnP structure, allowing a better dispersion in the polymer matrix. Nevertheless, Stenvall et al. (2013) found that the influence of barrel temperature profile was small in the ternary blend of recycled acrylonitrile butadiene-styrene copolymer (ABS - $40 \mathrm{wt} . \%$ ), high impact polystyrene (HIPS - $40 \mathrm{wt} . \%$ ), and prepared with the various polypropylene (PP-10 wt.\%).

On the other hand, the Young's modulus, as depicted in Fig. 2B, increased both when the hybrid nanofiller was introduced and when the temperature profile increased. At MT and HT profiles, Young's modulus increases by $25 \%$ and $18 \%$, respectively. Again, this finding is attributed to the dispersion of the GnP/C20A hybrid nanofiller in the PLA matrix. The higher aspect ratio and large surface area of both GnP and C20A results in this enhancement.

Based on previous studies, the addition of nanofillers to the polymer matrix will produce an increase of the mechanical properties of the nanocomposites (Bijarimi et al., 2019; Pluta et al., 2006). From this experiment, it was observed a significant change from PLA/ABS without nanofillers to the nanofillers, as shown in Fig. 2. The stress at the peak showed the high- est value at a high temperature, that is $43.1 \mathrm{MPa}$, compared to the other temperature conditions. However, it was also noted that, in the PLA/ABS binary blend, the value of stress at the peak for medium and high temperatures was not significantly different with the low temperature.

The elongation at break for the nanocomposites blends with different temperature conditions is shown in Table 3. It is observed that the addition of nanofillers increased the elongation at break by $20 \%, 4.7 \%$, and $33.3 \%$ for low, medium and high temperature with nanofillers loading, respectively, as shown in Fig. 2C. It was also observed that for PLA/ABS/GnP/C20A nanocomposites, the high temperature condition setting gave the highest elongation at break of $2.4 \%$. Both stress at peak and elongation at break were also observed to have significant increase from PLA/ABS to PLA/ABS/GnP/C20A, as shown in Fig. 2A, C. Figure 2B shows the Young's modulus trend for all nanocomposites, which was observed to have an increasing trend from PLA/ABS to PLA/ABS/GnP/C20A. This is probably due to adding the nanoclay and graphene to the polymer matrix; the brittleness characteristic of PLA, which has a high modulus (3800 MPa), has been improved. The incorporation of nanoclay also improves the Young's modulus of PLA/ ABS/GnP compared to PLA/ABS, due to hydrogen bonds in

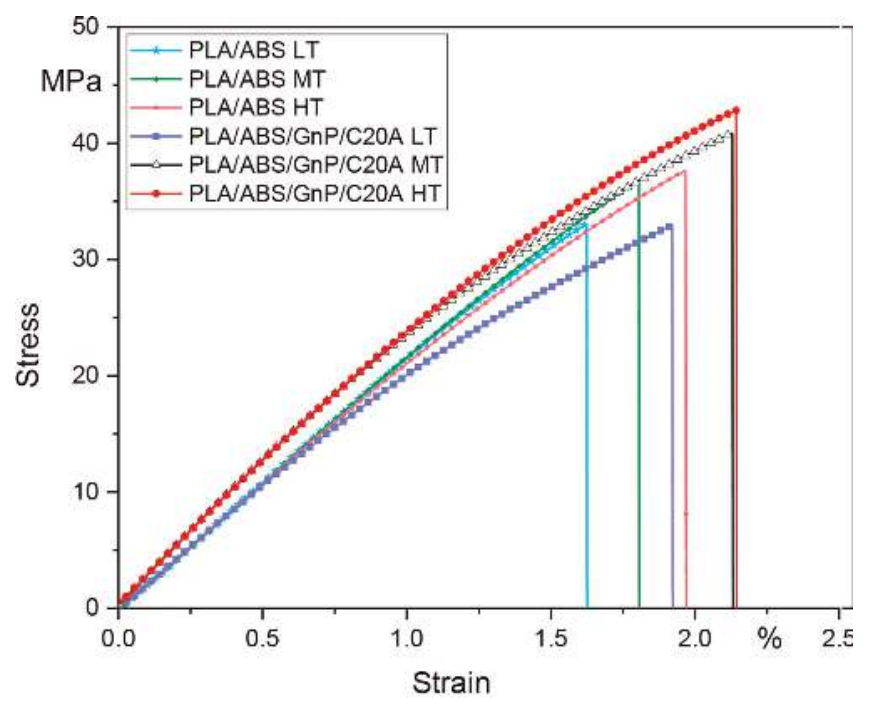

Fig. 1. Stress-strain curves of PLA/ABS and PLA/ABS/GnP/C20A across temperature profiles

\begin{tabular}{|c|c|c|c|c|}
\hline Composition & $\begin{array}{c}\text { Young's modulus } \\
\text { MPa }\end{array}$ & $\begin{array}{c}\text { Stress at peak } \\
\text { MPa }\end{array}$ & $\begin{array}{c}\text { Elongation at break } \\
\%\end{array}$ & $\begin{array}{c}\text { Toughness } \\
\mathrm{J} / \mathrm{m}^{3}\end{array}$ \\
\hline PLA/ABS LT & 1715 & 32.9 & 1.5 & 28.1 \\
PLA/ABS MT & 1999 & 34.9 & 2.1 & 34.7 \\
PLA/ABS HT & 2152 & 37.6 & 1.8 & 39.5 \\
PLA/ABS/GnP/C20A LT & 2335 & 34.4 & 2.2 & 35.2 \\
PLA/ABS/GnP/C20A MT & 2506 & 41.9 & 2.4 & 59.8 \\
PLA/ABS/GnP/C20A HT & 2533 & 43.1 & & 51.7 \\
\hline
\end{tabular}

Table 3. Mechanical properties of the PLA, ABS, PLA/ABS and PLA/ABS/GnP/C20A nanocomposites 


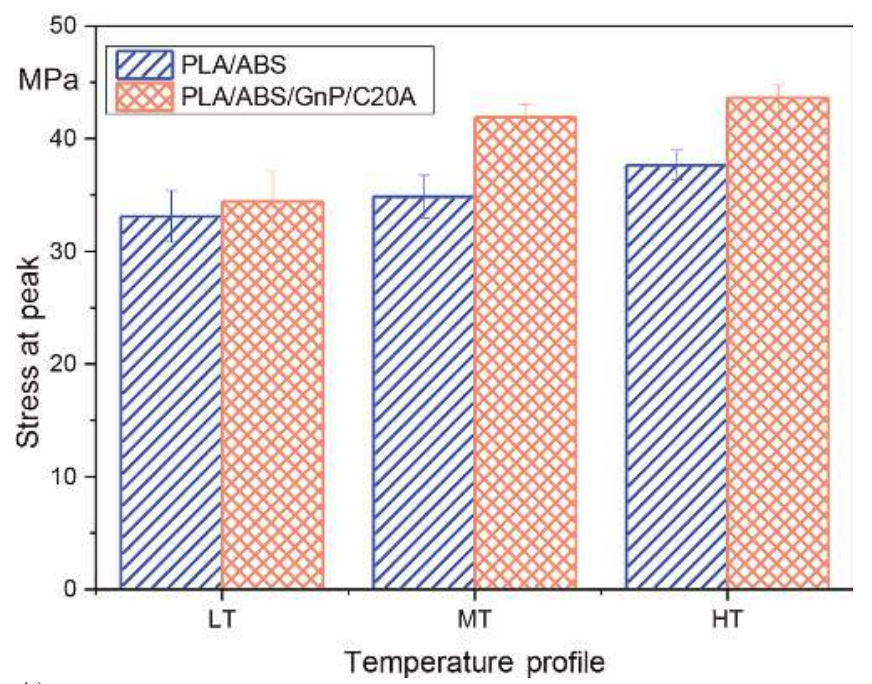

A)

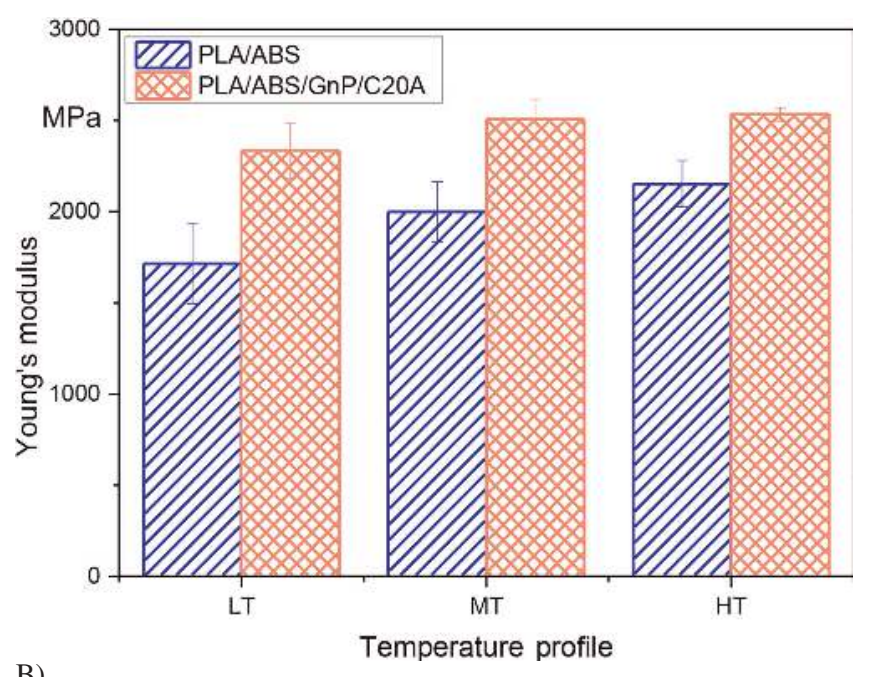

B)

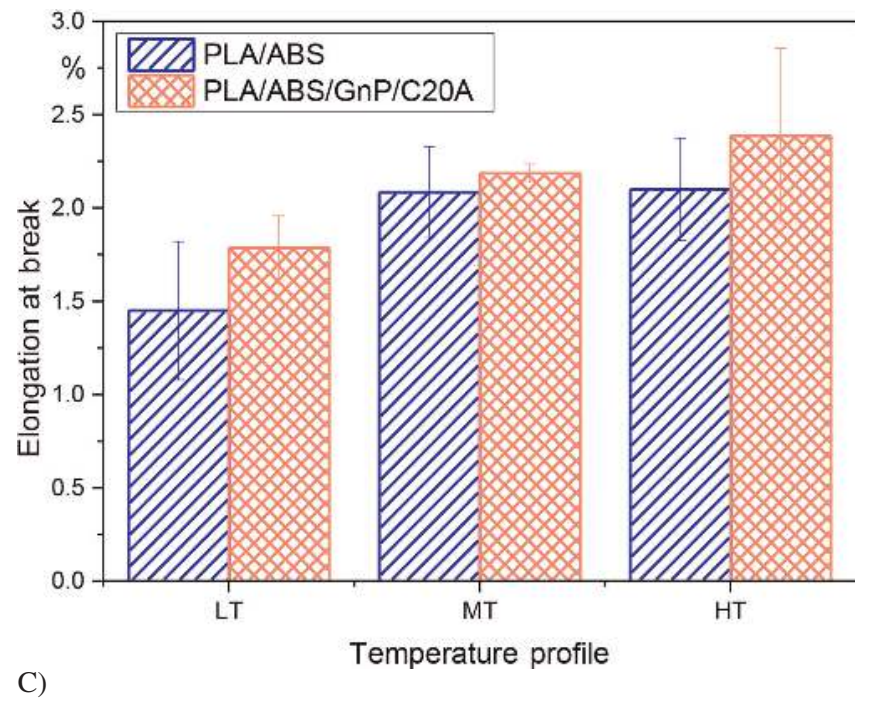

Fig. 2. Stress at peak (A), Young's modulus (B) and elongation at break $(C)$ of PLA/ABS and PLA/ABS/GnP/C20 nanocomposites as a function of the temperature profile the functional group on the surface of nanoclay with carbonyl groups of the PLA and the dispersion of nanoclay in the polymer matrix. In addition, it was also observed that toughness as measured by the energy absorbed before fracture progressed from the PLA/ABS to PLA/ABS/GnP/C20A MT blend systems. Similar findings were reported by Cao et al. (2010), who found that a small percentage of graphene contributed to a $26 \%$ increase in tensile strength and an $18 \%$ increase in Young's modulus.

\subsection{MMT/GNP Dispersion and Morphology}

It is known that fillers increase the tensile modulus, and heat deflection temperatures, and that this is governed by the size and shape of the filler particles. Figures 3 to 5 depict the SEM micrographs obtained from the surface of the tensile test fractured specimens of both PLA/ABS and PLA/ABS/GnP/C20A at all three temperature profiles. It can be seen that all the nanocomposites exhibit voids. Void formation indicates immiscibility and low interfacial adhesion of the polymer, as commented by Bouakaz et al. (2017).

From the micrographs, in PLA/ABS (Fig. 3A), the void sizes in the LT profile (average size of the void is $1.451 \mu \mathrm{m}$ ) are prominent and are much more uneven in comparison to those at the other temperatures. This suggests that the fracture occurred at the interface between the 4 phases of the polymer (ABS consisted of three polymers in the blends). On the other hand, a more uniform dispersion of PLA was observed in the MT and HT profiles of the PLA/ABS, as shown in Fig. 4 and 5. Also, the void size varies only slightly (average sizes of the voids of MT and HT are $0.533 \mu \mathrm{m}$ and $0.726 \mu \mathrm{m}$, respectively). These findings agree with the tensile results, in which the difference was quite small but increased with the decrease in the size of voids.

On the other hand, in the micrograph of PLA/ABS/GnP/ C20A (Fig. 3B), while the void sizes are similar (average size of the void of LT, MT, and HT are approximately $0.949 \mu \mathrm{m}$, $0.711 \mu \mathrm{m}$, and $0.976 \mu \mathrm{m}$, respectively) in all temperature profiles, the dispersion of the GnP varies. The dispersion of graphene can be noted by the graphitic layer structure seen in the micrograph (Gao et al., 2017). The more the morphology is layered, the higher the extent of agglomeration of the GnP. The agglomeration of the $\mathrm{GnP}$ is indicated in the LT profile by the highly layered and flaky surface seen in the micrograph. The agglomeration was quite severe, explaining the dramatic decrease in stress and elongation at break when the fillers were introduced in the LT profile in comparison to the neat PLA/ ABS nanocomposite, as evidenced from TEM microscopy in Fig. 6. However, as the processing temperature was increased, the dispersion improved (Ito et al., 2003). This could be attributed to the high Van der Waal forces between the GnP sheets. Increasing the temperature increases the kinetic energy required to break these forces and thus allows for better intercalation in the polymer matrices. 


\subsection{Fourier Transform Infrared (FTIR)}

Fourier transform infrared spectra for PLA, ABS, PLA/ABS, and $\mathrm{PLA} / \mathrm{ABS} / \mathrm{GnP} / \mathrm{C} 20 \mathrm{~A}$ at LT/MT/HT temperature profiles are displayed in Fig. 7. The functional group interactions of the PLA with ABS, GnP and C20A through the shift of the absorption peak in specific regions was monitored. The characteristic peaks of PLA were at 1746, 2995, 2946, and $1080 \mathrm{~cm}^{-1}$ which are the frequencies of $\mathrm{C}=\mathrm{O},-\mathrm{CH}_{3}$ asymmetric, $-\mathrm{CH}_{3}$ symmetric, and $\mathrm{C}-\mathrm{O}$ functional groups respectively. As for ABS, the characteristic frequency was at $2924 \mathrm{~cm}^{-1}$ for the asymmetric and symmetric $\mathrm{CH}_{2}$ stretching, $2237 \mathrm{~cm}^{-1}$ for the $\mathrm{C} \equiv \mathrm{N}$ stretch, as well as $966 \mathrm{~cm}^{-1}$ and $912 \mathrm{~cm}^{-1}$ for the out-of-plane C-H stretch of butadiene (Bijarimi et al., 2019; Chieng et al., 2014; Ma, et al. 2012).

Figures 7A to D depict the FTIR spectra of neat PLA, neat ABS, PLA/ABS LT, and PLA/ABS/GnP/C20A LT. The nanocomposites exhibit the similar peaks that a nanocomposite of PLA and ABS should have without any other new peaks ob- served. This suggests that no chemical reaction occurs between any of the functional groups, which means that the nanocomposites are pure physical blends and thus are immiscible. This finding agrees with Vadori et al. (2016), where ABS did not play a significant role in the reaction; however, the interaction between PLA and ABS was due to mechanical interaction.

However, it was also noted in Fig. 7C and D that increasing the processing temperature profile increases the intensity of the peaks. According to the Beer-Lambert's law (Swinehart, 1962), the intensity of the peak is related to either the concentration of the functional group or the thickness of the sample (thickness relates to the light path). Because the samples have a similar thickness, this could mean that the higher the processing temperature, the higher the concentration of the functional groups in the sample. This could be due to the void formation that occurs during sample moulding. On the other hand, the intensity difference when filler was introduced could be attributed to filler agglomeration.

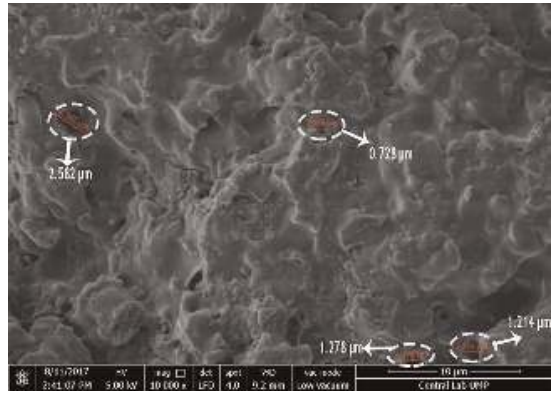

A)
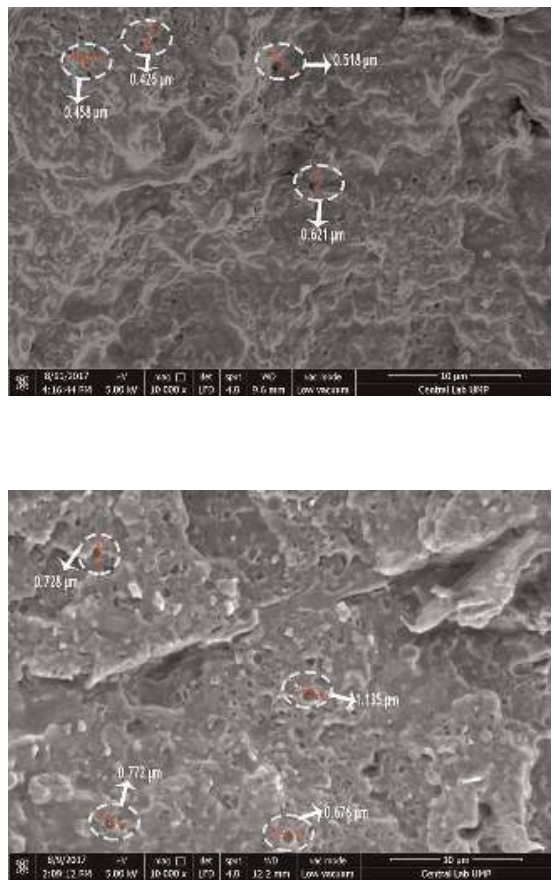

A)

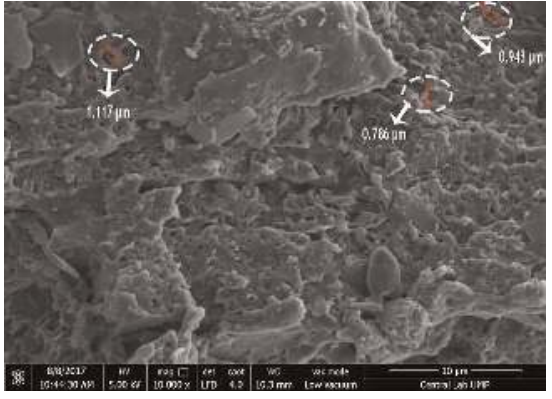

B)
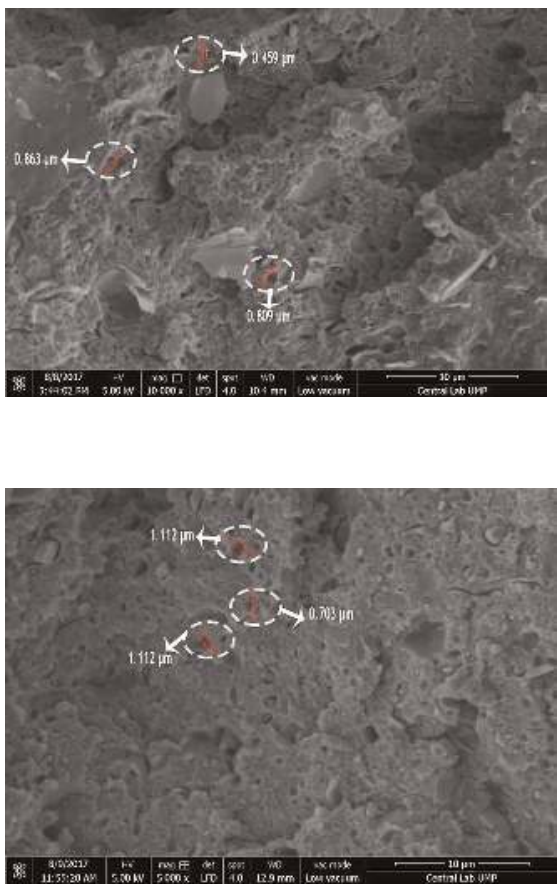

B)
Fig. 3. SEM micrograph taken from the surface of tensile fractured specimen at x10000 magnification for $L T$ profile, A) PLA/ ABS, B) PLA/ABS/GnP/C2OA

Fig. 4. SEM micrograph taken from the surface of tensile fractured specimen at x10000 magnification for MT profile, A) PLA/ $A B S, B) P L A / A B S / G n P / C 20 A$

Fig. 5. SEM micrograph taken from the surface of tensile fractured specimen at x10000 magnification for HT profile, A) PLA/ ABS, B) PLA/ABS/GnP/C2OA 


\subsection{Thermal Analysis}

Table 4 summarizes the thermal properties (glass transition temperature, melting temperature and cold crystallization) of the neat PLA, neat ABS, PLA/ABS, and PLA/ABS/GnP/ C20A nanocomposites obtained from DSC. Note that $\mathrm{T}_{\mathrm{g} 1}$ was not available for most of the polymers since the peak belonging to the butadiene component in the ABS was too close to the starting temperature. It was found that the nanocomposites possessed multiple $\mathrm{T}_{\mathrm{g}} \mathrm{s}$. This again indicates the immiscibility of the nanocomposite, which is in agreement with Fayt et al. (1985), who stated that immiscible polymer blends have multiple $T_{g}$ due to the phase separation of the polymers, resulting in low cohesive energy.

It was also noted that the melting temperature of the polymer blends was lower than that of the neat PLA and neat ABS. This could be due to a drop in the degree of crystallinity as a result of the amorphous nature of ABS. The degree of crystallinity affects the melting point of the polymer which agrees with findings of Wacharawichanant et al. (2017), who found that the melting point of PLA drops with the addition of propyleneethylene copolymer (PEC). They stated that this decrease in the melting point is due to the drop in the degree of crystallinity as a result of the amorphous nature of PEC. However, this could not be concluded with the lack of crystallinity data.

If the blend components are immiscible, the $\mathrm{T}_{\mathrm{g}}$ values of the individual polymers remain virtually unaffected. On the other hand, a completely miscible mixture would display a single $\mathrm{T}_{\mathrm{g}}$. As shown in Table 4, the cold crystallization temperature of PLA/ABS blends at the medium temperature was about $95.0^{\circ} \mathrm{C}$ and shifted to approximately $92.5^{\circ} \mathrm{C}$ after incorporation of the GnP. The combination effects of nucleation and diffusion-controlled growth processes resulted in the change of cold crystallization behavior in the PLA/ABS/GnP/C20A nanocomposites. It was observed that a slight change of PLA crystallinity after the addition of $\mathrm{GnP}$ did not induce a significant impact on the mechanical properties of the nanocomposites. However, the homogeneous dispersion of $\mathrm{GnP}$ in the polymer matrix and strong interactions between both components can influence the significant changes in the strength and modulus of PLA/ABS nanocomposites.

Tiwari and Natarajan (2008) observed that the $\mathrm{T}_{\mathrm{g}}$ of ABS hybrids remains unaffected by the incorporation of unmodified or organically modified montmorillonite (Cloisite 6A, 10A, $20 \mathrm{~A}$, and $25 \mathrm{~A}$ ) or laponite at $4 \%$ loading, independently of the particle and platelet size. Nevertheless, Yeh et al. (2006) found that the incorporation of organoclay resulted in an increase in $\mathrm{T}_{\mathrm{g}}$ in relation to that of neat $\mathrm{ABS}$ and interpreted this effect by the confinement of intercalated polymer chains within the organoclay galleries, which prevents the segmental motions of polymer chains.

The degradation behavior of PLA, ABS, PLA/ABS, and PLA/ABS/GnP/C20A nanocomposites are given in Fig. 8 and Table 5. The start of weight loss represents the onset temperature, while the end temperature is the temperature at the end of degradation. Since this is a blend of two polymers, the polymer blends result in having two degradation onset temperatures and peak temperatures. However, since the onset of the ABS occurs before PLA is fully degraded, the end temperature recorded is for full degradation of the polymer blends. In general, it was found that the degradation temperature of both PLA and ABS decreases when the polymer is blended, which is expected for a polymer blend. However, the addition of the fillers increases the thermal stability of the PLA/ABS nanocomposites, as depicted by the increase in onset temperature and peak temperature. This could be due to the nanoparticle's reinforcement

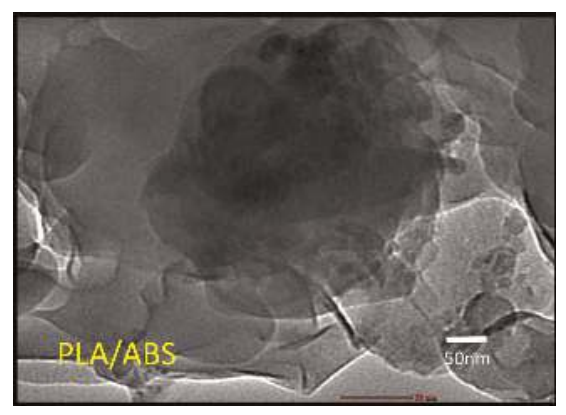

A)

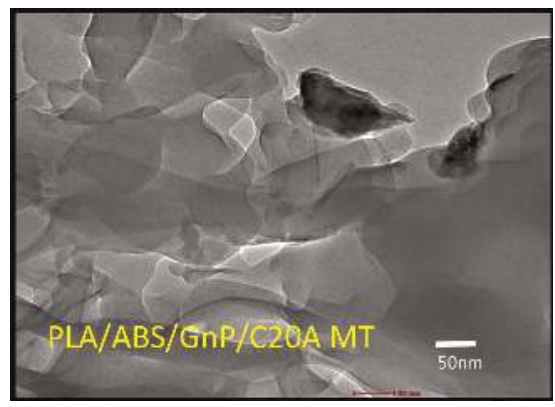

C)

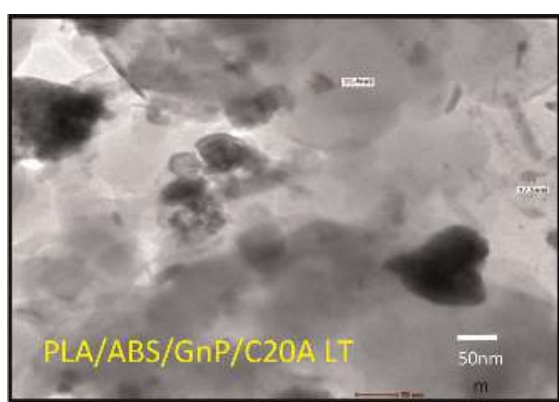

B)

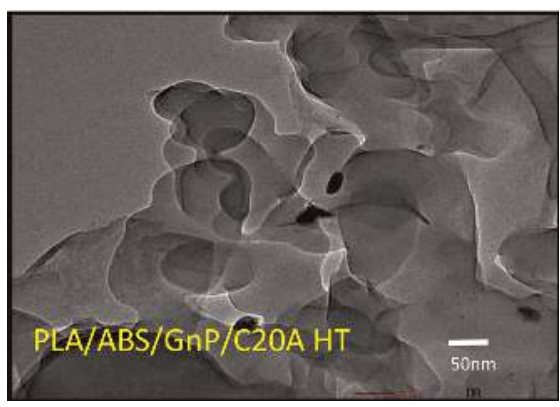

D)
Fig. 6. TEM images for (A) PLA/ABS profile, B) PLA/ABS/GnP/C20A LT profile, C) PLA/ ABS/GnP/C20MT profile, D) PLA/ABS/GnP/ C2OA HT profile 

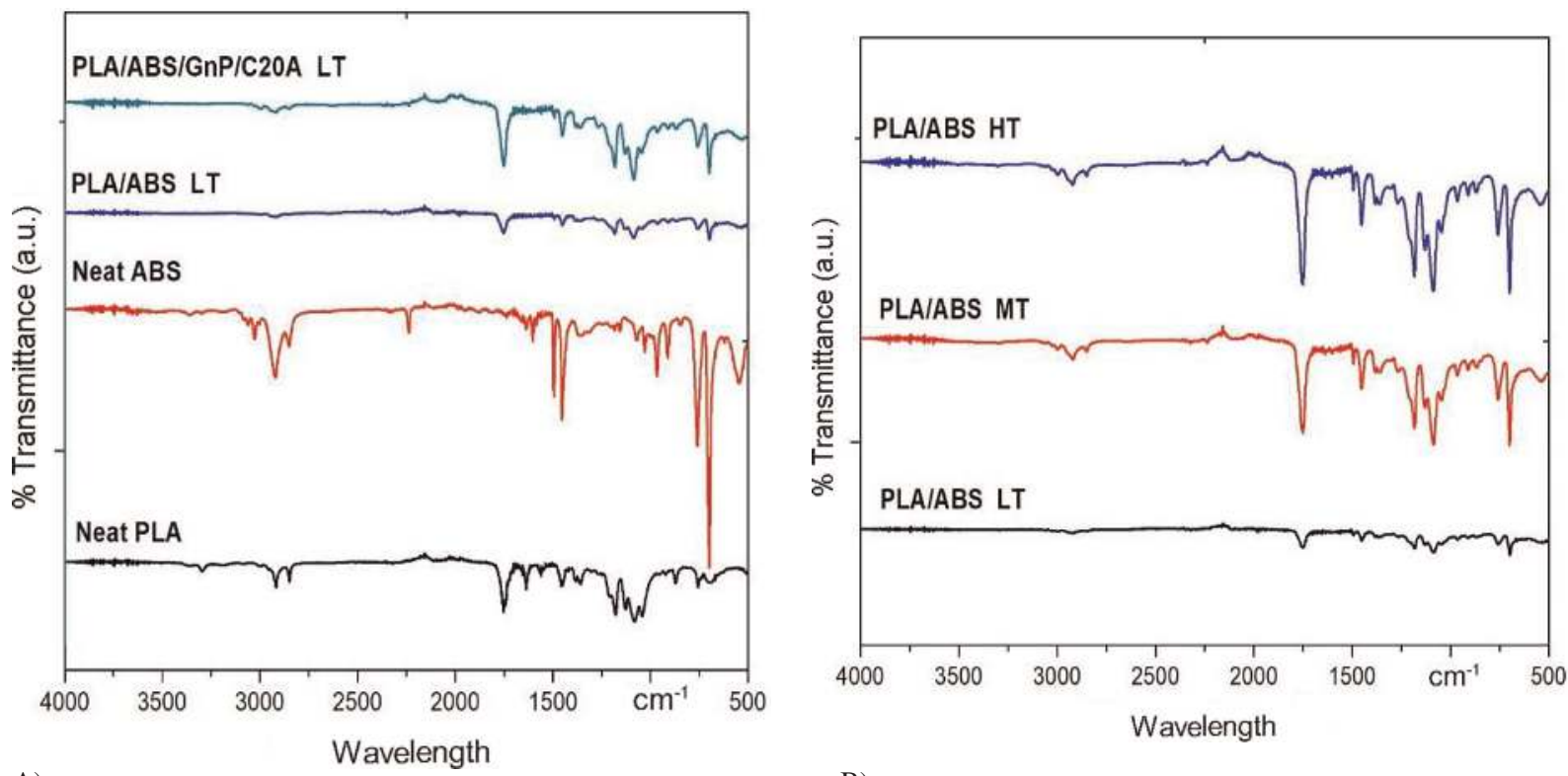

A)

B)
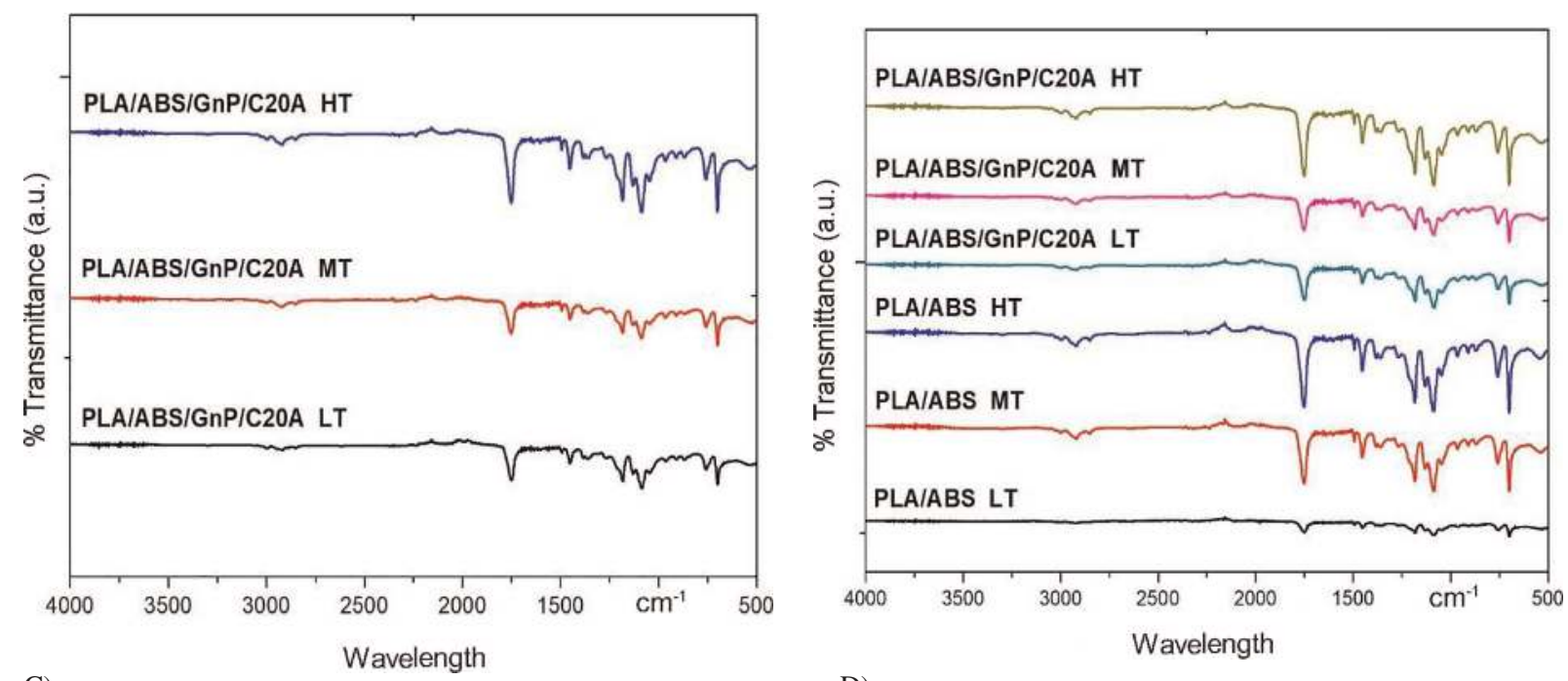

D)

Fig. 7. FTIR comparison for (A) neat PLA, neat ABS, PLA/ABS at low temperature profile and PLA/ABS/GnP/C20A at low temperature profile, B) PLA/ABS across temperature profiles, C) PLA/ABS/GnP/C20A across temperature profiles and (D) PLA/ABS and PLA/ABS/GnP/C20A across temperature profiles

\begin{tabular}{|c|c|c|c|}
\hline Composition & $\underset{\mathrm{C} 1}{\mathrm{~T}_{\mathrm{C} 1}}, \underset{\mathrm{g} 2}{\mathrm{~T}_{\mathrm{g}}}, \mathrm{T}_{\mathrm{g} 3}$ & $\begin{array}{l}\mathrm{T}_{\mathrm{c}} \\
{ }^{\circ} \mathrm{C}\end{array}$ & $\begin{array}{l}\mathrm{T}_{\mathrm{m}} \\
{ }^{\circ} \mathrm{C}\end{array}$ \\
\hline PLA & 60.0 & - & 170.2 \\
\hline PLA/ABS LT & $-, 62.5,150.0$ & 92.5 & 166.0 \\
\hline PLA/ABS MT & $-, 62.5,150.0$ & 95.0 & 165.8 \\
\hline PLA/ABS/GnP/C20A MT & $-, 60.0,150.0$ & 92.5 & 165.7 \\
\hline PLA/ABS/GnP/C20A HT & $-, 62.5,150.0$ & 92.5 & 165.9 \\
\hline
\end{tabular}

Table 4. DSC data of the neat PLA, neat ABS, PLA/ABS and PLA/ABS/GnP/C20A nanocomposites 
of the nanofillers, since both possess a higher decomposition temperature. This finding is in agreement with studies by Fukushima et al. (2010), who prepared PLA-based nanocomposites using two different nanofillers: expanded graphite and organically modified montmorillonite.

It can be seen that the decomposition of PLA started at $330.5^{\circ} \mathrm{C}$ and was completed at $368.6^{\circ} \mathrm{C}$ while the PLA/ABS blend at $\mathrm{HT}$ condition started to decompose at $315.4^{\circ} \mathrm{C}$ and was completed at $486.6^{\circ} \mathrm{C}$ and PLA/ABS/GnP/C20A blend at HT started to decompose at $337.9^{\circ} \mathrm{C}$ and completed at $482.5^{\circ} \mathrm{C}$. The result showed the onset of degradation of PLA/ ABS/GnP/C20A started slower than PLA and PLA/ABS binary blends. Thermograms of PLA/ABS/GnP/C20A HT showed higher onset degradation temperature than PLA/ABS HT due to the dispersion state of MMT layers in the polymer matrix.
The graphene improved the thermal stability of the PLA/ABS matrix owing to the intrinsic characteristic of the graphene. Graphene sheets take the role of thermal barriers and improve the thermal stability of the polymer matrix. The incorporation of $\mathrm{GnP}$ into the polymer matrix enhances the overall material's thermal stability. The layer structure gives a more significant barrier effect to inhibit the evaporation of the volatile degradation products generated during the thermal decomposition of the nanocomposite. This phenomenon was responsible for contributing to the enhancement for thermal stability for PLA/ ABS/GnP/C20A (Liang et al., 2009). It is worth noting that the compatibilizing action of $\mathrm{C} 20 \mathrm{~A}$ contributed to the decrease in the interfacial tension and hence leads to remarkable mechanical and thermal properties of the PLA/ABS/GnP/C20A nanocomposites. Figure 9 shows the XRD patterns for PLA/

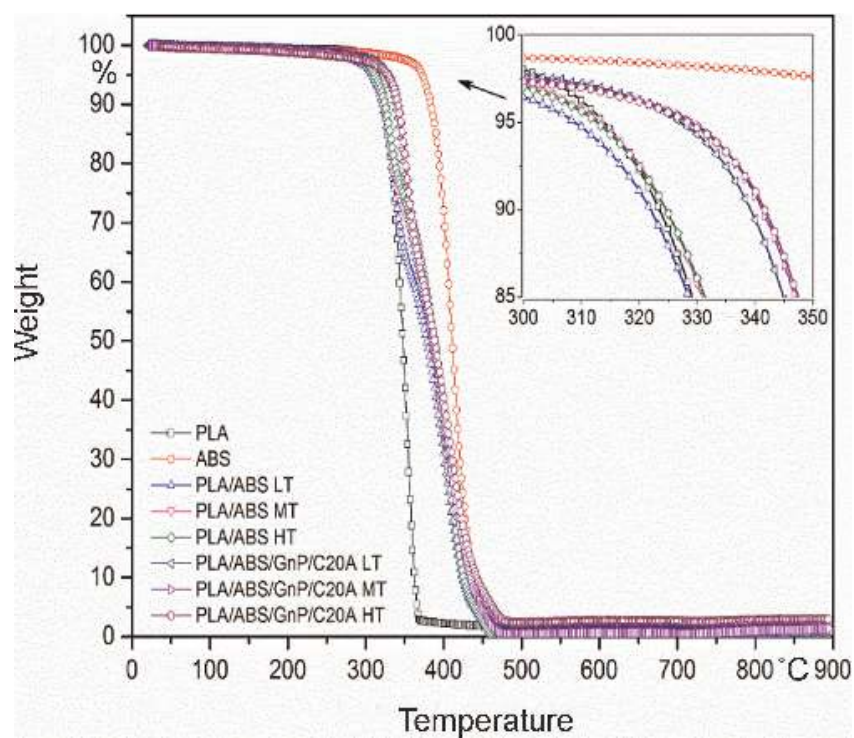

A)

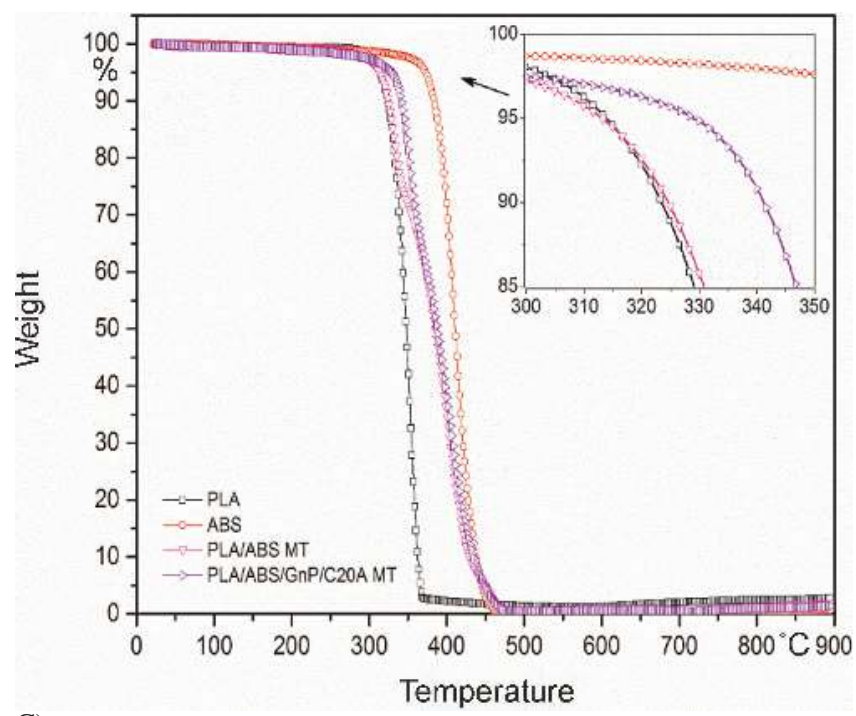

C)

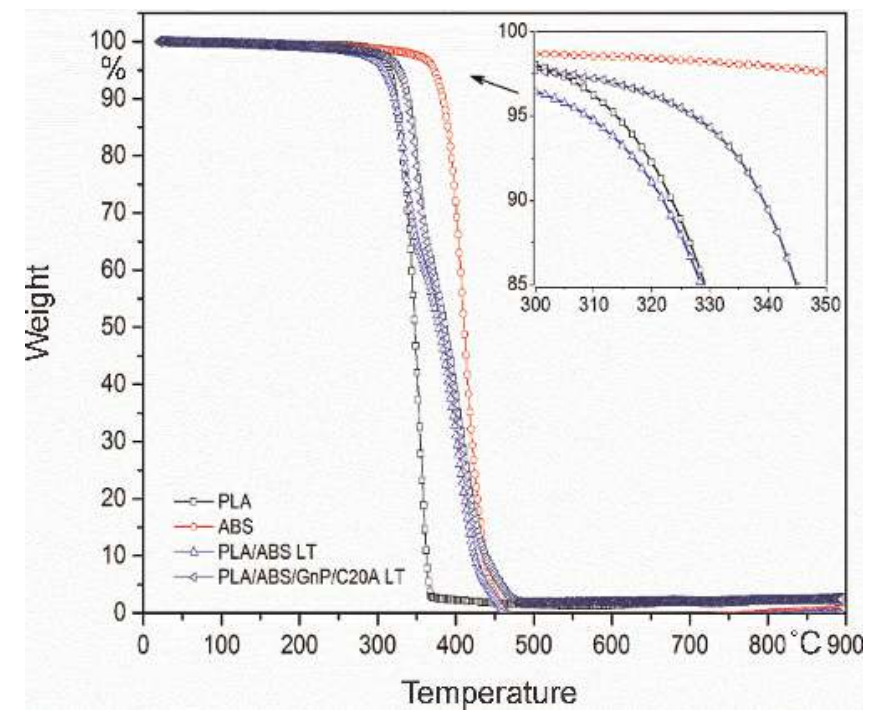

B)

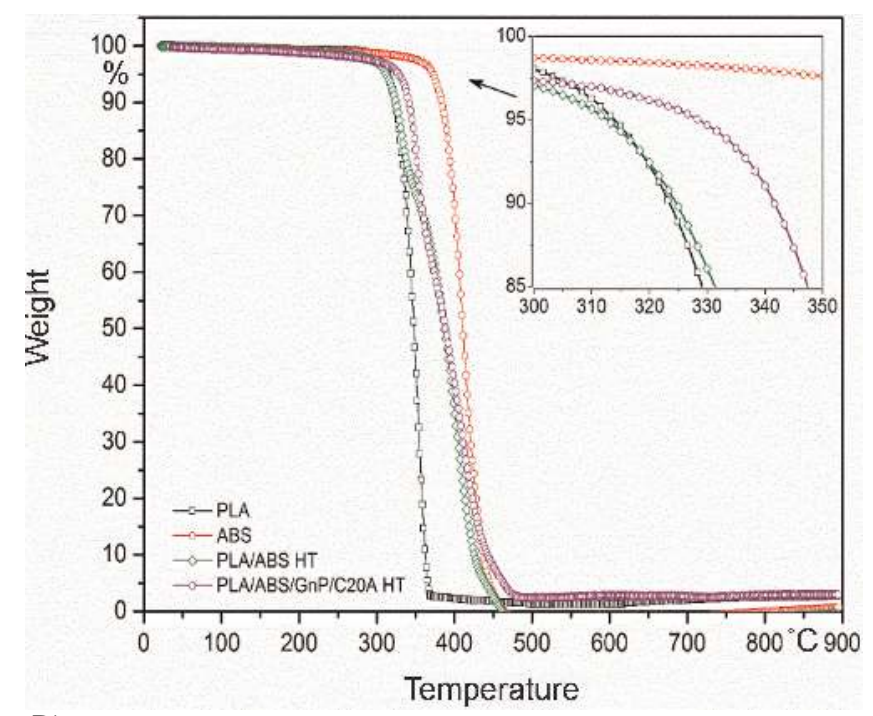

D)

Fig. 8. TGA curves of (A) neat PLA, neat ABS, PLA/ABS, and PLA/ABS/GnP/C20A nanocomposite, B) LT profile, C) MT profile and (D) HT profile 


\begin{tabular}{|c|c|c|c|}
\hline Composition & Onset temperature & $\begin{array}{c}\text { Peak temperature } \\
\text { C }\end{array}$ & $\begin{array}{c}\text { End temperature } \\
{ }^{\circ} \mathrm{C}\end{array}$ \\
\hline PLA & 330.5 & 352.9 & 368.6 \\
ABS & 388.5 & 410.6 & 464.3 \\
PLA/ABS LT & $317.0,379.6$ & $334.8,400.7$ & 476.5 \\
PLA/ABS MT & $316.5,376.7$ & $333.3,400.3$ & 491.5 \\
PLA/ABS HT & $315.4,377.2$ & $333.2,402.6$ & 483.9 \\
PLA/ABS/GnP/C20A LT & $334.3,387.2$ & $350.6,404.9$ & 482.6 \\
PLA/ABS/GnP/C20A MT & $336.0,390.0$ & $351.6,404.8$ & 482.5 \\
PLA/ABS/GnP/C20A HT & $337.9,389.5$ & $352.4,407.4$ & \\
\hline
\end{tabular}

Table 5. TGA data for neat PLA, neat ABS, PLA/ABS and PLA/ABS/GnP/C20A nanocomposites

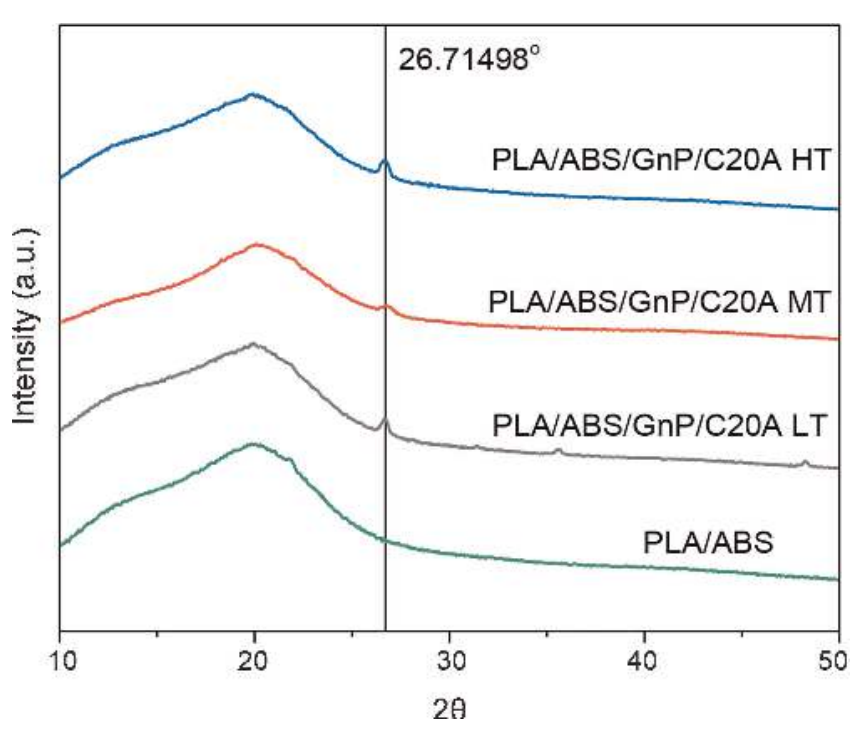

Fig. 9. XRD patterns for PLA/ABS and PLA/ABS/GnP/C20A for all temperature profiles

ABS and PLA/ABS/GnP/C20A for all temperature profiles. The peak at $26.71498^{\circ}$ is visible for the hybridized nanocomposites, as compared to a binary blend of PLA/ABS.

\section{Conclusions}

PLA/ABS/GnP/C20A nanocomposites were successfully prepared, and the effect of processing temperature profile on the mechanical, morphological, and thermal properties of PLA/ $\mathrm{ABS}$ and PLA/ABS/GnP/C20A nanocomposites were investigated. It was found that increasing the temperature profile enhances the mechanical properties of PLA/ABS/GnP/C20A, while no significant findings were observed in PLA/ABS. The tensile strength was observed to increase by $20 \%$ and $15 \%$ in the MT and HT profiles, respectively, while the Young's modulus increased by $25 \%$ and $18 \%$ in the MT and HT profiles, respectively. The enhancement of mechanical properties contributed by the dispersion of the fillers. Increasing the temperature results in better dispersion of the fillers, as can be seen from the morphological characterization by SEM and TEM. However, the FTIR results exhibited no significant changes, and no chemical reaction happens in any functional group, and DSC analysis also exhibits no significant changes under different temperature conditions.

In terms of thermal degradation analysis by TGA, the onset degradation temperature shows an increment of $22.5^{\circ} \mathrm{C}$ for PLA/ABS/GnP/C20A HT as compared to PLA/ABS HT binary blend.

\section{References}

Alexandre, M., Dubois, P., "Polymer-Layered Silicate Nanocomposites: Preparation, Properties and Uses of a New Class of Materials", Mater. Sci. Eng., R, 28, 1-63 (2000), DOI:10.1016/S0927-796X(00)00012-7

Arroyo, O. H., Huneault, M. A., Favis, B. D. and Bureau, M. N., "Processing and Properties of PLA/Thermoplastic Starch/Montmorillonite Nanocomposites", Polym. Compos., 31, 114-127 (2010), DOI:10.1002/pc.20774

Bijarimi, M., Ahmad, S. and Alam, A. K. M. M., "Toughening Effect of Liquid Natural Rubber on the Morphology and Thermo-Mechanical Properties of the Poly(lactic acid) Ternary Blend", Polymer Bull., 74, 3301 - 3317 (2017), DOI:10.1007/s00289-016-1889-7

Bijarimi, M., Ahmad, S. and Rasid, R., "Melt Blends of Poly(lactic acid)/Natural Rubber and Liquid Epoxidised Natural Rubber", J. Rubber Res., 17, 57-68 (2014)

Bijarimi, M., Amirul, M., Norazmi, M., Ramli, A., Desa, M. S. Z., Desa, M. A. and Abu Samah, M. A., "Preparation and Characterization of Poly(lactic acid) (PLA)/Polyamide 6 (PA6)/Graphene Nanoplatelet (GNP) Blends Bio-Based Nanocomposites", Mater. Res. Express, 6, 055044 (2019), DOI:10.1088/2053-1591/ab05a3

Bijarimi, M., Shahadah, N., Ramli, A., Nurdin, S., Alhadadi, W., Muzakkar, M. Z. and Jaafar, J., "Poly(lactic acid) (PLA)/Acrylonitrile Butadiene Styrene (ABS) with Graphene Nanoplatelet (GNP) Nanocomposites", Indonesian Journal of Chemistry, 20, 276-281 (2020), DOI: $10.22146 /$ ijc.40880

Bitinis, N., Verdejo, R., Maya, E. M., Espuche, E., Cassagnau, P. and Lopez-Manchado, M. A., "Physicochemical Properties of Organoclay Filled Polylactic Acid/Natural Rubber Blend Bionanocomposites", Compos. Sci. Technol., 72, 305-313 (2012), DOI:10.1016/j.compscitech.2011.11.018

Bouakaz, B. S., Habi, A., Grohens, Y. and Pillin, I., "Organomontmorillonite/Graphene-PLA/PCL Nanofilled Blends: New Strategy to Enhance the Functional Properties of PLA/PCL Blend", Appl. Clay Sci., 139, 81-91 (2017), DOI:10.1016/j.clay.2017.01.014

Bouakaz, B. S., Pillin, I., Habi, A. and Grohens, Y., "Synergy between Fillers in Organomontmorillonite/Graphene-PLA Nanocomposites", Appl. Clay Sci., 116-117, 69-77 (2015), DOI:10.1016/j.clay.2015.08.017 
Cao, Y., Feng, J. and Wu, P., "Preparation of Organically Dispersible Graphene Nanosheet Powders through a Lyophilization Method and their Poly(lactic acid) Composites", Carbon, 48, 3834-3839 (2010), DOI:10.1016/j.carbon.2010.06.048

Chieng, B., Ibrahim, N., Yunus, W., Hussein, M., Then, Y. and Loo, Y., "Effects of Graphene Nanoplatelets and Reduced Graphene Oxide on Poly(lactic acid) and Plasticized Poly(lactic acid): A Comparative Study", Polymers, 6, 2232-2232 (2014), DOI:10.3390/polym6082232

Choe, I.-J., Lee, J. H., Yu, J. H. and Yoon, J.-S., "Mechanical Properties of Acrylonitrile-Butadiene-Styrene Copolymer/Poly(L-lactic acid) Blends and their Composites", J. Appl. Polym. Sci., 131, 40329 (2014), DOI:10.1002/app.40329

Coiai, S., Cicogna, F., De Santi, A., Pérez Amaro, L., Spiniello, R., Signori, F., Fiori, S., Oberhauser, W. and Passaglia, E., "MMT and LDH Organo-Modification with Surfactants Tailored for PLA Nanocomposites", eXPRESS Polym. Lett., 11, 163-175 (2017), DOI:10.3144/expresspolymlett.2017.18

Dong, W., He, M., Wang, H., Ren, F., Zhang, J., Zhao, X. and Li, Y., "PLLA/ABS Blends Compatibilized by Reactive Comb Polymers: Double Tg Depression and Significantly Improved Toughness", ACS Sustainable Chem. Eng., 3, 2542-2550 (2015), DOI: $10.1021 /$ acssuschemeng. 5 b00740

Fayt, R., Hadjiandreou, P. and Teyssie, P., "Molecular Design of Multicomponent Polymer Systems. VII. Emulsifying Effect of Poly(ethylene-B-styrene) Copolymer in High-Density Polyethylene/ Polystyrene Blends", J. Polym. Sci., Part A: Polym. Chem., 23, 337-342 (1985), DOI:10.1002/pol.1985.170230209

Fu, Y., Liu, L., Zhang, J. and Hiscox, W. C., "Functionalized Graphenes with Polymer Toughener as Novel Interface Modifier for Property-Tailored Polylactic Acid/Graphene Nanocomposites", Polymer, 55, 6381-6389 (2014), DOI:10.1016/j.polymer.2014.10.014

Fukushima, K., Murariu, M., Camino, G. and Dubois, P., "Effect of Expanded Graphite/Layered-Silicate Clay on Thermal, Mechanical and Fire Retardant Properties of Poly(lactic acid)", Polym. Degrad. Stab., 95, 1063-1076 (2010),

DOI:10.1016/j.polymdegradstab.2010.02.029

Gao, Y., Picot, O. T., Bilotti, E. and Peijs, T., "Influence of Filler Size on the Properties of Poly(lactic acid) (PLA)/Graphene Nanoplatelet (GNP) Nanocomposites", Eur. Polym. J., 86, 117-131 (2017), DOI:10.1016/j.eurpolymj.2016.10.045

Gonçalves, C., Pinto, A., Machado, A. V., Moreira, J., Gonçalves, I. C. and Magalhães, F., "Biocompatible Reinforcement of Poly(lactic acid) with Graphene Nanoplatelets", Polym. Compos., 39, E308E320 (2016), DOI:10.1002/pc.24050

Ito, E. N., Pessan, L. A., Covas, J. A. and Hage, E., "Analysis of the Morphological Development of PBT/ABS Blends during the Twin Screw Extrusion and Injection Molding Processes", Int. Polym. Proc., 18, 376-381 (2003), DOI:10.3139/217.1780

Jalalvandi, E., Majid, R., Ghanbari, T. and Ilbeygi, H., "Effects of Montmorillonite (MMT) on Morphological, Tensile, Physical Barrier Properties and Biodegradability of Polylactic Acid/Starch/ MMT Nanocomposites", J. Thermoplast. Compos. Mater., 28, 496-509 (2015), DOI:10.1177/0892705713486129

Keramati, M., Ghasemi, I., Karrabi, M., Azizi, H. and Sabzi, M., "Incorporation of Surface Modified Graphene Nanoplatelets for Development of Shape Memory PLA Nanocomposite", Fibers Polym., 17, 1062-1068 (2016), DOI:10.1007/s12221-016-6329-7

Li, S., Li, Z., Burnett, T. L., Slater, T. J. A., Hashimoto, T. and Young, R. J., "Nanocomposites of Graphene Nanoplatelets in Natural Rubber: Microstructure and Mechanisms of Reinforcement", J. Mater. Sci., 52, 9558 - 9572 (2017), DOI:10.1007/s10853-017-1144-0

Li, Y., Shimizu, H., "Improvement in Toughness of Poly(L-lactide) (PLLA) through Reactive Blending with Acrylonitrile-ButadieneStyrene Copolymer (ABS): Morphology and Properties", Eur. Polym. J., 45, 738-746 (2009), DOI:10.1016/j.eurpolymj.2008.12.010

Liang, J., Huang, Y., Zhang, L., Wang, Y., Ma, Y., Cuo, T. and Chen, Y., "Molecular-Level Dispersion of Graphene into Poly(vinyl alcohol) and Effective Reinforcement of their Nanocomposites", Adv. Funct. Mater., 19, 2297-2302 (2009),

DOI:10.1002/adfm.200801776
Ma, H., Wang, J. and Fang, Z., "Cross-Linking of a Novel Reactive Polymeric Intumescent Flame Retardant to ABS Copolymer and its Flame Retardancy Properties", Polym. Degrad. Stab., 97, 1596-1605 (2012), DOI:10.1016/j.polymdegradstab.2012.06.030

Manafi, P., Ghasemi, I., Karrabi, M., Azizi, H. and Ehsaninamin, P., "Effect of Graphene Nanoplatelets on Crystallization Kinetics of Poly(lactic acid)", Soft Mater., 12, 433-444 (2014), DOI: $10.1080 / 1539445 X .2014 .959598$

Nofar, M., Sacligil, D., Carreau, P. J., Kamal, M. R. and Heuzey, M.-C., "Poly(lactic acid) Blends: Processing, Properties and Applications", Int. J. Biol. Macromol., 125, 307-360 (2019), PMid:30528997; DOI:10.1016/j.ijbiomac.2018.12.002

Pinto, A. M., Gonçalves, C., Gonçalves, I. C. and Magalhães, F. D., "Effect of Biodegradation on Thermo-Mechanical Properties and Biocompatibility of Poly(lactic acid)/Graphene Nanoplatelets Composites", Eur. Polym. J., 85, 431 -444 (2016), DOI:10.1016/j.eurpolymj.2016.10.046

Pluta, M., Paul, M. A., Alexandre, M. and Dubois, P., "Plasticized Polylactide/Clay Nanocomposites. I. The Role of Filler Content and its Surface Organo-Modification on the Physico-Chemical Properties", J. Polym. Sci., Part B: Polym. Phys., 44, 299-311 (2006), DOI:10.1002/polb.20694

Sabzi, M., Jiang, L. and Nikfarjam, N., "Graphene Nanoplatelets as Rheology Modifiers for Polylactic Acid: Graphene Aspect-RatioDependent Nonlinear Rheological Behavior", Ind. Eng. Chem. Res., 54, 8175-8182 (2015), DOI:10.1021/acs.iecr.5b01863

Scaffaro, R., Botta, L., Maio, A. and Gallo, G., "PLA Graphene Nanoplatelets Nanocomposites: Physical Properties and Release Kinetics of an Antimicrobial Agent", Composites Part B, 109, 138-146 (2017), DOI:10.1016/j.compositesb.2016.10.058

Scaffaro, R., Botta, L., Maio, A., Mistretta, M. C. and La Mantia, F. P., "Effect of Graphene Nanoplatelets on the Physical and Antimicrobial Properties of Biopolymer-Based Nanocomposites", Materials, 9, 351 (2016), DOI:10.3390/ma9050351

Sharma, N., Alam, S. N., Ray, B. C., Yadav, S. and Biswas, K., "SilicaGraphene Nanoplatelets and Silica-MWCNT Composites: Microstructure and Mechanical Properties", Diamond Relat. Mater., 87, 186-201 (2018), DOI:10.1016/j.diamond.2018.06.009

Stenvall, E., Tostar, S., Boldizar, A. and Foreman, M. R. S. J., "The Influence of Extrusion Conditions on Mechanical and Thermal Properties of Virgin and Recycled PP, HIPS, ABS and their Ternary Blends", Int. Polym. Proc., 28, 341-549 (2013), DOI: $10.3139 / 217.2801$

Swinehart, D. F., "The Beer-Lambert Law", J. Chem. Educ., 39, $333-$ 335 (1962), DOI:10.1021/ed039p333

Tiwari, R. R., Natarajan, U., "Effect of Organic Modifiers and Silicate Type on Filler Dispersion, Thermal, and Mechanical Properties of ABS-Clay Nanocomposites", J. Appl. Polym. Sci., 110, 23742383 (2008), DOI:10.1002/app.28699

Vadori, R., Misra, M. and Mohanty, A. K., "Sustainable Biobased Blends from the Reactive Extrusion of Polylactide and Acrylonitrile Butadiene Styrene”, J. Appl. Polym. Sci., 133, 43771 (2016), DOI:10.1002/app.43771

Vadori, R., Misra, M. and Mohanty, A. K., "Statistical Optimization of Compatibilized Blends of Poly(lactic acid) and Acrylonitrile Butadiene Styrene", J. Appl. Polym. Sci., 134, 44516 (2017), DOI: $10.1002 /$ app. 44516

Wacharawichanant, S., Ounyai, C. and Rassamee, P., "Effects of Organoclay to Miscibility, Mechanical and Thermal Properties of Poly (lactic acid) and Propylene-Ethylene Copolymer Blends", IOP Conf. Ser.: Mater. Sci. Eng., 223, 012016 (2017),

DOI: $10.1088 / 1757-899 X / 223 / 1 / 012016$

Wu, N., Zhang, H., "Toughening of Poly(L-lactide) Modified by a Small Amount of Acrylonitrile-Butadiene-Styrene Core-Shell Copolymer", J. Appl. Polym. Sci., 132, 42554 (2015), DOI: $10.1002 / a p p .42554$

Yeh, J. M., Chen, C. L., Huang, C. C., Chang, F. C., Chen, S. C., Su, P. L., Kuo, C. C., Hsu, J. T., Chen, B. and Yu, Y. H., "Effect of Organoclay on the Thermal Stability, Mechanical Strength, and Surface Wettability of Injection-Molded ABS-Clay Nanocomposite Materials Prepared by Melt Intercalation", J. Appl. Polym. Sci., 99, 1576-1582 (2006), DOI:10.1002/app.22329 
M. Bijarimi et al.: PLA/ABS/GnP/C20A Nanocomposites: Effect of Processing Temperatures

Young, R. J., Liu, M., Kinloch, I. A., Li, S., Zhao, X., Vallés, C. and Papageorgiou, D. G., "The Mechanics of Reinforcement of Polymers by Graphene Nanoplatelets", Compos. Sci. Technol., 154, 110-116 (2018), DOI:10.1016/j.compscitech.2017.11.007

\section{Acknowledgements}

The authors would like to thank the Universiti Malaysia Pahang (UMP) for sponsoring this research project under a grant FRGS/1/2019/TK05/UMP/02/1 (RDU1901104) and RDU190380.
Date received: January 12, 2020

Date accepted: May 02, 2020

Bibliography

DOI 10.3139/217.3934

Intern. Polymer Processing

XXXV (2020) 4; page 355-366

(c) Carl Hanser Verlag $\mathrm{GmbH} \&$ Co. KG

ISSN 0930-777X 